\title{
A RESEARCH PROGRAM TO MAP THE GALACTIC EMISSION AT LOW FREQUENCIES ${ }^{1}$
}

\author{
G. De Amici ${ }^{a}$, S. Torres ${ }^{b}$, M. Bensadoun ${ }^{a}$, G. Dall'Oglio ${ }^{c}$, \\ M. Limon ${ }^{a}$, G. Sironi ${ }^{d}$, G. Smoot ${ }^{a}$, T. Villela ${ }^{e}$, C. Witebsky ${ }^{a}$ \\ ${ }^{a}$ Space Sciences Laboratory and Lawrence Berkeley Laboratory, UC Berkeley, USA \\ ${ }^{b}$ Universidad de los Andes, and Centro Internacional de Física, Colombia \\ "Università di Roma, "La Sapienza", Italy. \\ ${ }^{d}$ Università degli Studi, Milano, Italy. \\ ${ }^{e} \mathrm{INPE} / \mathrm{CNPq}$, Brazil.
}

\begin{abstract}
The need for accurate and complete maps of the galaxy at radio frequencies is made evident not only by the importance of the data in the study of galactic dynamics and structure formation but also in the analysis of cosmic background radiation data. A summary of the existing radio surveys of our galaxy is presented along with their limitations. A new program, the Galactic Emission Mapping (GEM) project, to obtain calibrated and complete maps of the galaxy in the 400 to $1400 \mathrm{MHz}$ range, is outlined.
\end{abstract}

\section{Introduction}

The systematic study of the continuous radio emission allows a direct estimate of some of the basic parameters that explain the dynamics and formation of the galaxy such as: the temperature and density of the interstellar clouds, the spectral index of the energy spectrum of cosmic ray electrons, and the mean intensity of the galactic magnetic field.

Complete radio maps of the galaxy are useful not only for galactic studies, but they are also required in the analysis and interpretation of the recently discovered (Smoot et al. 1992) anisotropies of the cosmic microwave background (CMB) as well as on the measurements of its spectral distribution (Banday et al. 1991, Bensadoun et al. 1993).

In the centimeter to meter range of wavelengths the main contribution to the galactic signals comes from synchrotron radiation of relativistic electrons in weak interstellar magnetic fields, and thermal Bremsstrahlung produced by free-free transitions of electrons in the electric fields of ions (HII). The observed brightness temperature of these three components has a spectral profile which can be expressed by a power law, $T(\nu) \sim \nu^{\alpha}$, with spectral index $\alpha=-2.75,-2.1$ and 1.5 for the synchrotron (Rybicki \& Lightman, 1979), HII (Scheffler \& Elsässer 1987) and dust emission (Wright et al. 1991) respectively (Figure 1).

The thermal radiation of dust clouds is the main component at the high end of the radio spectrum and in the IR region and is negligible for all observations below about $50 \mathrm{GHz}$.

\footnotetext{
${ }^{1}$ Presented by S. Torres at the Second United Nations/European Space Agency Workshop on Basic Space Science, San José and Bogotá, 2-13 November, 1992.
} 
At intermediate frequencies the thermal HII emission is dominant, especially in the plane of the galaxy $\left(|b|<5^{\circ}\right.$ ), where giant gas clouds provide the material and the conditions for large concentrations of ionized hydrogen. At the low-frequency end of the spectrum and away from the galactic plane, the synchrotron radiation is dominant.

The need for complete and accurately calibrated maps of the galactic emission is well established. Here we describe a strategy to survey the radio continuum, in the 400 to 1400 $\mathrm{MHz}$ range, from different sites around the globe in order to achieve full sky coverage.

\section{Synchrotron Continuum}

The non-thermal component of the galactic background is interpreted as produced by the energy loss of cosmic ray electrons with relativistic velocities, when their trajectories are deflected around the field lines of the interstellar magnetic field. The radiation is linearly polarized and its intensity depends on the number density of relativistic electrons along the line of sight, while the spectral index depends on the energy spectrum of the electrons, and the intensity of the magnetic field. At a given frequency $\nu$, the measured brightness temperature is:

$$
T_{\nu} \sim \nu^{-2} \int \varepsilon_{\mathrm{Syn}}(\nu) d l
$$

with the integral taken along the line-of-sight. The emission coefficient, or radiation energy emitted per unit of volume, time, frequency and solid angle, for synchrotron radiation is:

$$
\varepsilon_{\text {syn }} \propto B_{\perp}^{(\gamma+1) / 2} \nu^{-(\gamma-1) / 2} .
$$

The term $B_{\perp}$ denotes the perpendicular component of the magnetic field along the line-of-sight, and $\gamma$ is the spectral index in the differential energy spectrum of cosmic ray electrons: $N(E) d E \propto E^{-\gamma} d E$. From (2) the connection between the spectral indexes of the synchrotron brightness temperature spectral profile and the cosmic ray differential energy distribution, $\alpha=-(\gamma+3) / 2$, is evident. Thus, with an estimate of the effective magnetic field, the total energy density of the electrons can be calculated. Conversely, if the total energy density of the electrons is measured directly, an estimate of the effective galactic magnetic field can be obtained. The measured value of $\gamma \approx 2.5$ gives the known spectral index for galactic synchrotron radiation $\alpha \approx-2.75$ (Rybicki \& Lightman, 1979).

Any accurate estimate of the contribution of the synchrotron signal to the overall sky brightness at mid- and high- frequencies (where the synchrotron signal is smaller than the CMB, but still not negligible) depends on a precise set of absolutely calibrated measurements at low frequencies (where the synchrotron signal is dominant), so as to ascertain the zero level of the galactic background and its spectral index as a function of direction.

\section{Thermal Continuum}

The interaction of free electrons with positive ions results in the emission of radiation whose intensity depends on the electron velocity and the distance of closest approach. Under the assumption that the energy of the electrons undergoes only small changes, which is correct for Bremstrahlung-like encounters, the electrons can be assumed to have a Maxwell distribution of energies. For a cloud of hydrogen-like ions of charge $Z e$ the emission coefficient 
is (Scheffler \& Elsässer 1987):

$$
\varepsilon_{f f} \sim \frac{Z^{2} g}{T^{1 / 2}} N_{e} N_{i} \exp (-h \nu / k T)
$$

where $T$ is the temperature of the cloud, $N_{e}$ and $N_{i}$ the electron and positive ion number density, $h$ Plank's constant, $k$ Boltzmann constant, and $g$, the Gaunt factor, which in the radio region is approximately $g \sim T^{0.15} \nu^{-0.1}$.

In the limiting case of an optically thin layer the intensity of the radiation $I_{\nu}$ is proportional to the emission coefficient, $I_{\nu} \sim T^{-0.35} \nu^{-0.1}$, thus the brightness temperature is:

$$
T_{\nu}=\left(\frac{c^{2}}{2 \nu^{2} k}\right) I_{\nu} \sim \nu^{-2.1} T^{-0.35},
$$

which shows how for the temperatures of typical HII regions $T \approx 10^{4}$, emission occurs essentially only in the radio and IR part of the spectrum. If the temperature of the HII clouds is known, then from the brightness temperature it is possible to derive a mean value of the electron density of the cloud.

\section{The existing surveys}

The only full-sky survey at the present (Haslam et al. 1982) is at $408 \mathrm{MHz}$ (figure 2). Partial surveys exist at frequencies below $5 \mathrm{GHz}$; at $1420 \mathrm{MHz}$, Reich et al. (1986) account for just the northern celestial hemisphere, above -19 deg declination. Other surveys at 38 (Milogradov-Turin 1984); 178 (Turtle and Baldwin 1962); 404 (Pauliny-Toth and Shakeshaft 1962); 610 (Webster 1974) and 820 (Berkhuijsen 1972) $\mathrm{MHz}$ have similarly incomplete sky coverage, and the missing observations always affect the southern sky. Another survey, which will cover the southern celestial sky at $2300 \mathrm{MHz}$, is under way (Jonas et al. priv. comm.) Table 1 summarizes the characteristics of these survey; the $2300 \mathrm{MHz}$ map is still unpublished, and is not included in this summary, while the survey of discrete sources at $1400 \mathrm{MHz}$ (Condon et al. 1986) are biased to exclude the diffuse continuum.

The existing maps have excellent angular resolution (up to $0.8^{\circ}$ ) but several shortcomings arise when they have to be used to determine the spectral index and absolute level of sky radiation from a given area. It has been exhaustively shown (Lawson et al. 1987) how the published results of these surveys are mutually incompatible, and large corrections need to be made in order to reconcile their quoted values. In fact, the relative gain uncertainty for the 408 and $1420 \mathrm{MHz}$ surveys is 10 and $5 \%$, respectively, and the absolute calibration of the maps is determined by comparison with absolute measurements that are over 20 years old. This translates into a $3 \mathrm{~K}$ uncertainty for the $408 \mathrm{MHz}$ map, and a $0.5 \mathrm{~K}$ uncertainty for the $1420 \mathrm{MHz}$ map. It should also be noted that the map by Haslam does not agree with the absolute calibrated measurement of the South Celestial Pole conducted at the same frequency by Price (1969). Even after introducing these corrections, however, the relative size of the error bars are so large that the spectral index is very poorly determined for all frequency ranges, and totally uncertain beyond $820 \mathrm{MHz}$.

From the summary presented above the importance of acquiring a new, accurate, calibrated and consistent set of data is evident. The remainder of this pajer presents the the experimental design that we will use to complete such a set of maps. 


\section{The Galactic Emission Mapping (GEM) Project}

An international collaboration (presently Brazil, Colombia, Italy, and the US, but open to new partners) has been set up to conduct measurements of the absolute sky temperature in the frequency range above $400 \mathrm{MHz}$. In order to improve upon the existing knowledge, the new measurements will have full-sky coverage, $5^{\circ}$ resolution or smaller, and absolute temperature calibration to $1 \mathrm{~K}$ at $408 \mathrm{MHz}$, and better than $0.1 \mathrm{~K}$ at $1400 \mathrm{MHz}$ and above. These requirements place conflicting demands on the design of the experiment. Parabolic reflector antennas with suitable resolution can be made steerable and relatively compact, but they are difficult to calibrate due to spillover, sidelobes, and diffraction by the feed assembly, or secondary reflector, and its supporting structure. In contrast, the sidelobe characteristics and insertion loss of a suitable horn antenna can be determined to the necessary level of accuracy from theory and measurement, but such an antenna is large, cumbersome to operate, and difficult to steer. To resolve these conflicts, we plan to use a parabolic reflector antenna to produce the full-sky maps and a conical horn to determine the absolute temperature scale. The parabolic antenna consists of a $5.5 \mathrm{~m}$ reflector with a feed antenna at the primary focus. Several receivers will be used, each one optimized for one of the different frequency bands. The parabola can be disassembled in small pieces for easy transportation to several sites on both hemispheres.

The systematic errors that appear in the existing maps, in part due to the fact that they were made with different instruments from different sites, will be minimized by using the same instrument, or a clone, at different sites. The minimum number of sites/instruments is defined by the local horizon and by the mechanical quality of the dish antennas used for the measurements. If the zenith angle is limited to a $30^{\circ}$ angle, the mechanical deformation of the parabolic surface is negligible, the contribution of the surrounding structures and terrain through the antenna sidelobes can be controlled with ground shields, and the antenna mount is of relatively simple construction. Figure 3 shows the part of the sky that can be observed by an instrument which is tilted up to $30^{\circ}$ from the local zenith. We have considered sites at several possible latitudes. While the apparent section of the sky seen from each site is approximately constant, the real solid angle observed changes with latitude, permitting the widest coverage and access to the most interesting targets to observers located near the Equator. On the other hand, the sites nearest to the Poles are expected to produce the most accurate calibrations, since the celestial poles can be observed and their signal repeatedly compared to a reference external load for any length of time, and with minimal movements of the antennas.

The best possible approach is to have several observing sites, from where a moderate size antenna can be used to observe a significant part of the sky. The observing sites must be chosen so that substantial overlapping exists between each target area. This will allow us to require that only a few points in the sky (e.g. the poles) be measured with absolute accuracy.

The absolute calibration of the maps will be determined by comparing the antenna temperature of the sky against the antenna temperature of external targets. Internal consistency will be achieved by a noise source fired at regular intervals during the observing run. Details of the calibration strategy are discussed by De Amici et al. (1993).

The receiver, which measures the total intensity of the emission, will consists of a direct RF-gain and total power front end. The signal collected by the antenna is filtered and 
amplified, rectified and detected, fed to a DC amplifier, and finally integrated, resulting in a very simple and straightforward receiver layout.

Measurements of the radio interference (RFI) level at some of the sites considered for the measurements show that even in remote areas the radio frequency spectrum is not free from interfering signals (Torres et al. 1993). In order to deal with the high RFI levels, the use of a correlator unit of suitable bandwidth has been foreseen. In a correlator, the signal is downconverted to a very low frequency, and the correlator is used to identify unwanted interference, which shows up as narrow lines. The data are then purged of RFI during analysis, digitized and processed by the on-line data acquisition system.

\section{Site Selection}

Starting from the South, an ideal distribution of locations includes sites spaced about 40 degrees apart in latitude. A few of these sites have already been identified. Two sites are available in Antarctica: the South Pole station $\left(90^{\circ} \mathrm{S}\right)$ and Terra Nova Bay $\left(75^{\circ} \mathrm{S}\right)$ (Dall'Oglio et al. 1992.) At northern mid-latitudes $\left(\approx 38^{\circ} \mathrm{N}\right)$ the California Institute of Technology maintains the Owens Valley Radio Observatory and the University of California the Hat Creek Radio Observatory, as well as the White Mountain Research Station (these sites are within driving distance from the home campus of the University of California at Berkeley).

The search for a site near the Equator is in progress. Hoeneisen et al. (1992) have identified six sites in Colombia and one site in Ecuador; these are located between $0^{\circ}$ and $<5^{\circ} \mathrm{N}$, and at altitudes from $2,600 \mathrm{~m}$ to $5,200 \mathrm{~m}$. Being located in a tropical region, the issue of weather may be a matter of concern. However, due to the presence of three mountain ranges (in the case of Colombia) and a complex system of atmospheric circulation, the weather conditions exhibit a pattern characterized by two rain/dry periods during a year, allowing for at least two windows of favorable dry weather six months apart. Of the six sites studied by Hoeneisen et al. the Chimborazo mountain $\left(4,000 \mathrm{~m}, 2^{\circ} \mathrm{S}\right)$ in Ecuador and Villa de Leyva $\left(3,400 \mathrm{~m}, 5^{\circ} \mathrm{N}\right)$ in Colombia offer the best infrastructure conditions. A complementary site search and RFI measurement expedition (Torres et al. 1993) has evaluated two additional sites: Ubaté $(2,500 \mathrm{~m})$ at $150 \mathrm{Km}$ from Bogotá, and Chía at 40 $\mathrm{Km}$ from Bogotá. RFI measurements on these sites have shown that mountain peaks near metropolitan areas are exposed to high levels of RFI, and a compromise must be accepted. We are considering sites located in shallow valleys, such as the Ubaté site.

Unfortunately, the elevation of the observing site is an important parameter in the selection process. As the thickness of the atmospheric layer above the antenna increases, so does the atmospheric signal, which is caused by the wings of the emission lines of water at $22 \mathrm{GHz}$ and oxygen at $60 \mathrm{GHz}$. The oxygen component is usually very stable and well mixed and under conditions of good weather accounts for 70-95\% (depending on the site altitude) of the signal at the GEM frequencies. Its contribution can be reliably modelled from theory. The water component, on the contrary, is highly variable and even in clear weather a passing cloud can change the antenna temperature of the atmosphere by 10 $20 \%$. As time and instrument conditions allow, we will use the parabolic antenna to track selected parts of the sky as they cross the meridian, to verify the cosecant dependence of the atmospheric signal on the zenith angle (Witebsky et al. 1986). Since most of the existing data sets have been measured in conditions of temperate climates, a series of measurements 
in tropical environment would be an important contribution to the studies of atmospheric propagation.

\section{Acknowledgments}

This work has been supported by the US Department of Energy under contract number DEAC03-76SF00098, by the US National Science Foundation under grant \# FD 91-02295, and by Colciencias of Colombia, project \# 1204-05-012-91. We have drawn from the expertise and gratefully acknowledge the collaboration of J. Aymon, M. Bersanelli, H. Dougherty, D. Heine, and W. Vinje.

\section{References}

Banday, A. J. and Wolfendale, A. W. 1991, MNRAS, 248, 705

Bensadoun, M., et al. 1993, ApJ, 409, 1

Berkhuijsen, E. M. 1972, Astr. \& Astp., 24, 143

Condon, J. and Broderick, J. 1986, Ap J. Suppl., 91, 1051

Dall'Oglio, G., et al. 1992, Experimental Astronomy, 2, 275

De Amici, G., et al. 1993, in: S. Holt (edt.) Back to the Galaxy, (New York: AIP conf. proc.), in press

Haslam, C. G. T., et al. 1982, Astr. \& Astp. Suppl., 47, 1

Hoeneisen, B., et al. 1992, in: M. Boratav, J. W. Croning, A. A. Watson (edts.) Cosmic rays above $10^{19}$ eV, Nuc. Phys. B (proc. suppl.) $28 \mathrm{~B}, 191$

Lawson, K. D., et al. 1987, MNRAS, 225, 307

Milogradov-Turin, J. 1984, MNRAS, 161, 269

Pauliny-Toth, I. I. K. and Shakeshaft, J. R. 1962, MNRAS, 124, 61

Price, R. M. 1969, Aust. J. Phys., 22, 641

Reich, P., et al. 1986, Astr. \& Astp. Suppl., 63, 205

Rybicki, G.B. and Lightman, A.P. 1979, Radiative Processes in Astrophysics (New York: Wiley \& Sons)

Scheffler, H. and Elsässer, H. 1987, Physics of the Galaxy and Interstellar Matter (New York: Springer-Verlag)

Smoot, G. F., et al. 1992, ApJ, 396, L1

Torres, S., et al. 1993, LBL-preprint No.34161

Turtle, A. J. and Baldwin, J. E. 1962, MNRAS, 124, 36

Webster, A. S. 1974, MNRAS, 166, 355

Witebsky, C., et al. 1986, ApJ, 310, 145

Wrigth, E. L., et al. 1991, ApJ, 381, 200 
Table 1 - Summary of the available radio maps.

\begin{tabular}{|c|c|c|c|c|c|c|}
\hline $\begin{array}{c}\text { frequency } \\
(\mathrm{MHz})\end{array}$ & $\begin{array}{c}\text { HPBW } \\
(\mathrm{deg})\end{array}$ & $\begin{array}{c}\text { Gain } \\
\text { uncertainty } \\
(\%)\end{array}$ & $\begin{array}{c}\text { zero level } \\
\text { uncertainty } \\
(\%)\end{array}$ & $\begin{array}{c}\text { zero level } \\
\text { uncertainty } \\
(\mathrm{K})\end{array}$ & $\begin{array}{c}\text { zero level } \\
\text { correction } \\
(\mathrm{K})\end{array}$ & $\begin{array}{c}\text { sky } \\
\text { coverage } \\
(\%)\end{array}$ \\
\hline 38 & 7.5 & 5 & 4 & 300 & 0 & 72 \\
178 & $0.22 \times 5$ & 10 & 15 & 15 & $+27.5^{a}$ & 57 \\
404 & $8.5 \times 6.5$ & 3.6 & 25 & 2.3 & $\mathrm{~N} / \mathrm{A}$ & $68^{b}$ \\
408 & 0.85 & 10 & 33 & 3 & $0^{a}$ & 100 \\
610 & 15 & 4 & $c$ & $c$ & $\mathrm{~N} / \mathrm{A}$ & $<50^{b}$ \\
820 & 1.2 & 6 & 40 & 0.6 & $-0.97^{a}$ & 57 \\
1420 & 0.6 & 5 & 100 & 0.5 & $-0.13^{a}$ & 68 \\
\hline
\end{tabular}

$\left({ }^{a}\right)$ as determined by Lawson et al. 1987

$\left({ }^{b}\right)$ the sky was undersampled at the resolution of the survey

(c) this survey did not attempt to measure the zero level of the data

\section{Figure Captions}

Figure 1- Frequency dependence of the antenna temperature of the Galactic emission; the synchrotron emission has been scaled with a power law slowly varying from -2.75 to -3.2 ; the Hir with a 2.1 power law; the dust component with a 1.5 power law. Typical values of intensity inside and outside of the galactic plane are shown for the synchrotron and HII components. The signal from the dust component away from the galactic plane falls outside the temperature scale shown here.

Figure 2- The radio emission of the galaxy at $408 \mathrm{MHz}$.

Figure 3- Several different sites, disseminated from the north to the south, will be needed to cover the whole celestial sphere. A few potential sites have been identified in this figure, together with the part of the sky that is observable by an instrument tilted to no more than $30 \mathrm{deg}$ from zenith. An overview of the sites distribution along the N-S direction: an artic site $\left(70^{\circ} \mathrm{N}\right)$, White Mountain (California, $38^{\circ} \mathrm{N}$ ), Bogotá (Colombia, $4^{\circ} \mathrm{N}$ ), Porto Alegre (Brazil, $30^{\circ} \mathrm{S}$ ), Terra Nova Bay (Antarctica, $75^{\circ} \mathrm{S}$ ). 


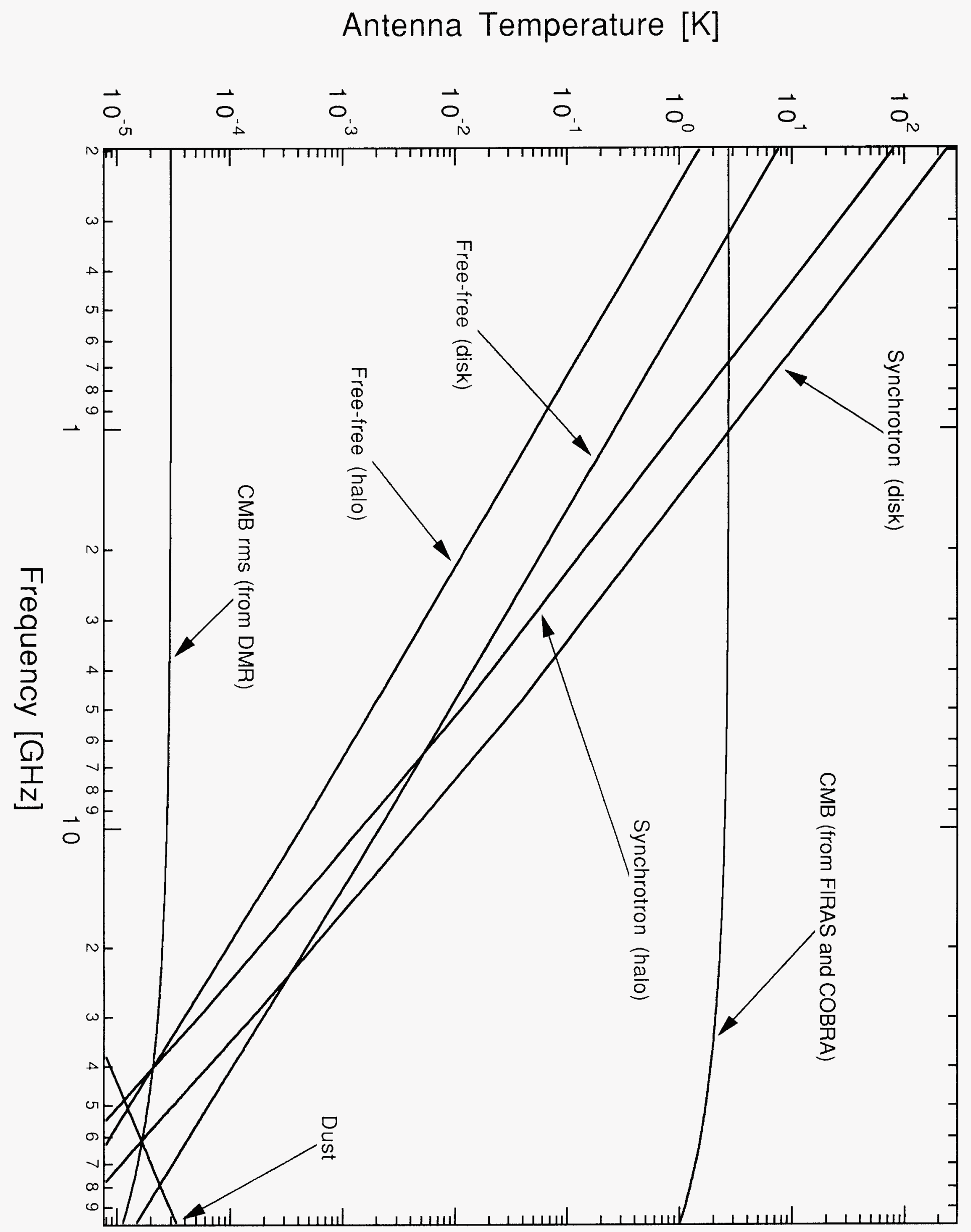




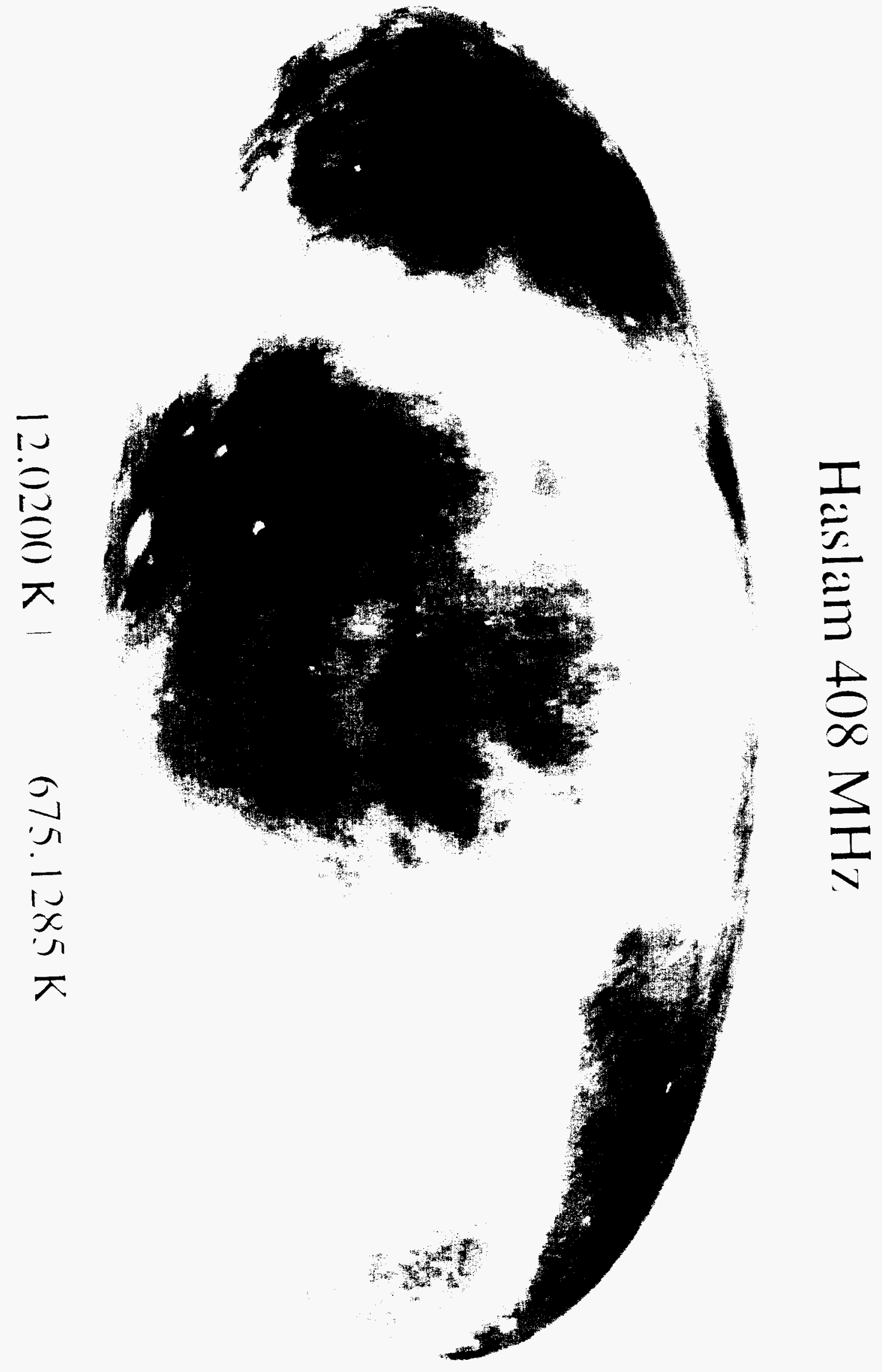




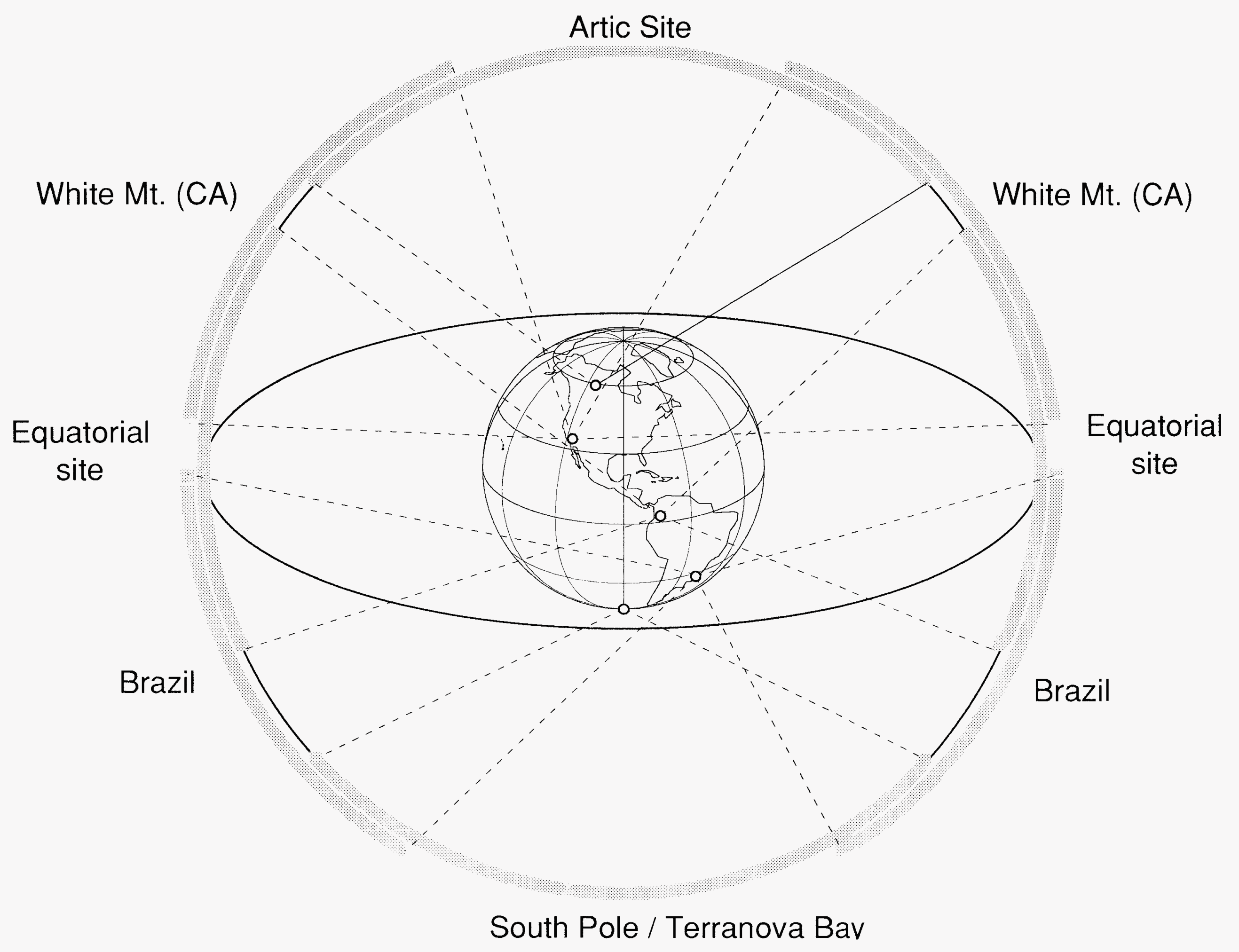

